\title{
Pragmatische Lösungen zur Bewirtschaftung des Leistungskatalogs
}

\author{
Im Rahmen des Projekts SwissHTA haben Vertreter der Krankenversicher, der \\ Pharmaindustrie und der Ärzteschaft ein Konsenspapier über die Anwen- \\ dung von Health Technology Assessment (HTA) erarbeitet. Sie schlagen \\ pragmatische Lösungen zur Bewirtschaftung des Leistungskatalogs vor.
}

Thomas Cueni

Generalsekretär Interpharma
Chronische Krankheiten werden künftig massiv zunehmen und zur grossen Herausforderung für das Gesundheitswesen und die Volkswirtschaft werden. Wirksame Prävention und rechtzeitige Behandlung vermindern nicht nur Leid der Betroffenen, sondern können auch Kosten im Gesundheitswesen und noch bedeutend höhere volkswirtschaftliche Kosten reduzieren. Gleichzeitig stehen nicht unbegrenzt Mittel für Investitionen in die Gesundheit zur Verfügung. Alle Beteiligten im Gesundheitswesen werden sich deshalb der Diskussion über «Value for Money», d. h. über Kosten und Nutzen von medizinischen Leistungen, noch stärker stellen müssen als heute. Rationierung und fixe Kostenschwellen sind keine Lösungen für die Schweiz. Sie treffen gerade diejenigen Patienten, die unsere Solidarität am meisten brauchen. Vielmehr gilt es, das Potential von HTA zu nutzen, um ein nachhaltig finanzierbares, qualitativ hochstehendes Gesundheitswesen zu erhalten. So gilt es, sowohl die Bewertung von Innovationen zu verbessern als auch bestehende Leistungen in Frage zu stellen.
Dies hat die Pharmaindustrie und die Krankenversicherer im Frühjahr 2010 veranlasst, das Projekt «Swiss HTA» zu initiieren. Etwas später sind FMH und SAMW sowie das Bundesamt für Gesundheit (BAG) als Beobachter dazugestossen und haben während eines Jahrs in einem intensiven Diskussionsprozess ein Konsenspapier über die Weiterentwicklung von HTA in der Schweiz erarbeitet. Dieser Konsensvorschlag zur Weiterentwicklung von HTA in der Schweiz ist von den beteiligten Organisationen - FMH, SAMW, santésuisse und Interpharma - formal verabschiedet und Verantwortlichen bei Bund und Kantonen zur Kenntnis gebracht worden. HTA soll demnach pragmatisch und schrittweise entwickelt werden und auf dem bestehenden System aufbauen. Die Führungsrolle kommt dabei dem Bund zu.

\section{Kein unüberlegtes «Copy-paste»}

SwissHTA hat die Entwicklung von HTA im Ausland studiert und ist zum Schluss gekommen, dass ausländische Modelle nicht einfach auf die Schweiz übertragbar sind. Die Beteiligten an SwissHTA sind 
sich aber einig, dass unser Land von internationalen Erfahrungen profitieren kann. Besonders intensiv diskutiert werden die Methoden zur Bewertung des Kosten-Nutzen-Verhältnisses. Fixe Schwellenwerte und die Abstützung auf eine einzige Methode (z.B. QALYs = quality adjusted life years) werden als ungeeignet und aufgrund ihrer Vereinfachungen Ungerechtigkeiten schaffend betrachtet. Dies heisst nicht, dass es keine Grenzsetzung für die Erstattungsfähigkeit von Leistungen geben soll. Diese soll jedoch aufgrund einer Methoden-offenen Wirtschaftlichkeitsbewertung aufgrund von transparenten WZW-Kriterien erfolgen. Vorgängig soll jedoch eine differenzierte Betrachtung der Wirksamkeit, d.h. des therapeutischen Mehrnutzens, erfolgen. Der Mehrnutzen neuer Therapien gegenüber dem Standard of Care soll unter Berücksichtigung der Signifikanz, der Relevanz, der Grösse des therapeutischen Effekts sowie des Vertrauens in die Evidenz in transparenten Nutzenkategorien bewertet werden.

\section{HTA in allen Leistungsbereichen}

Die Beteiligten von SwissHTA erwarten, dass HTA in allen Leistungsbereichen eingesetzt wird, also sowohl bei neuen Leistungen als auch bei bestehenden Leistungen. Obwohl das Krankenversicherungsgesetz bereits heute den Nachweis und die regelmässige Überprüfung der Wirksamkeit, der Zweckmässigkeit und der Wirtschaftlichkeit, der sogenannten WZW-Kriterien, für alle Leistungen verlangt, findet eine systematische Überprüfung eigentlich nur bei Medikamenten statt. Viele an- dere Leistungen mit unterschiedlicher Qualität und Effizienz werden indessen gar nie geprüft.

\section{Wissenschaftlich fundierte Bewertung für WZW}

Das grösste Potential zur Rationalisierung haben dabei bestehende Leistungen. Würde HTA nur bei neuen Technologien eingesetzt, würde dies die lückenhafte Umsetzung der WZW-Kriterien noch verschärfen. Konkret empfehlen sie zwei separate Verfahren: für die Bewertung neuer Leistungen einerseits «rapid HTAs» (rHTA) und für bestehende Technologien andererseits «complete HTAs» (cHTA). Bei beiden Verfahren sollen die WZW-Kriterien durch eine schrittweise und wissenschaftlich fundierte Bewertung operationalisiert werden. Innovationen können im rHTA-Verfahren rascher in den Leistungskatalog aufgenommen werden, aber mit grösserer Verbindlichkeit als heute überprüft werden. cHTA bietet die Chance, Qualität und Kosteneffizienz von komplexen Leistungen mit hohen Kostenfolgen für Gesundheitswesen und Volkswirtschaft zu verbessern. Eine Priorisierung für den Einsatz von cHTA bei bestehenden Leistungen durch den Bund unter Einbezug der Stakeholder wird als dringlich erachtet.

Die Projektträger von SwissHTA wollen den Bund weiterhin aktiv unterstützen und die Weiterentwicklung von HTA in der Schweiz mitgestalten. Den Beteiligten ist es besonders wichtig, die weitere Entwicklung von HTA in der Schweiz durch den konsequenten Einbezug der Stakeholder abzustützen. Nur so können akzeptable und akzeptierte Lösungen resultieren. 